\title{
Theoretical insights into bacterial chemotaxis
}

\author{
Marcus J. Tindall, 1,2,3* Eamonn A. Gaffney, ${ }^{4}$ Philip K. Maini ${ }^{4,5}$ \\ and Judith P. Armitage 5
}

Research into understanding bacterial chemotactic systems has become a paradigm for Systems Biology. Experimental and theoretical researchers have worked handin-hand for over 40 years to understand the intricate behavior driving bacterial species, in particular how such small creatures, usually not more than $5 \mu \mathrm{m}$ in length, detect and respond to small changes in their extracellular environment. In this review we highlight the importance that theoretical modeling has played in providing new insight and understanding into bacterial chemotaxis. We begin with an overview of the bacterial chemotaxis sensory response, before reviewing the role of theoretical modeling in understanding elements of the system on the single cell scale and features underpinning multiscale extensions to population models. ๑ 2012 Wiley Periodicals, Inc.

How to cite this article:
WIREs Syst Biol Med 2012, 4:247-259. doi: 10.1002/wsbm.1168

\section{BASICS OF BACTERIAL CHEMOTAXIS}

C hemotactic bacteria are usually small rod-like shaped cells with hemispherical ends, on an average between 2 and $5 \mu \mathrm{m}$ in length. They use complex intracellular response systems as they are too small to sense changes in their biochemical environment along the length of their body. Of the estimated many millions of bacterial species which are assumed to exist in nature, ${ }^{1}$ less than 100 have been studied in detail. Escherichia coli is the most widely studied chemotactic bacterium, but other species studied include Bacillus subtilis, Pseudomonas aeruginosa, and Rhodobacter sphaeroides.

To detect and respond to their environment, chemotactic bacteria rely upon three main physiological components:

\footnotetext{
*Correspondence to: m.tindall@reading.ac.uk

${ }^{1}$ School of Biological Sciences, University of Reading, Whiteknights, Reading, UK

${ }^{2}$ Department of Mathematics and Statistics, University of Reading, Whiteknights, Reading, UK

${ }^{3}$ Institute for Cardiovascular and Metabolic Research, University of Reading, Whiteknights, Reading, UK

${ }^{4}$ Centre for Mathematical Biology, Mathematical Institute, Oxford, UK

${ }^{5}$ Department of Biochemistry, Oxford Centre for Integrative Systems Biology, Oxford, UK
}

1. Clustered arrays of membrane spanning methyl accepting chemotaxis proteins (MCPs). These receptors are found at the polar ends of the cell;

2. Rotary molecular motors which drive helical flagella; and

3. An internal intracellular biochemical signaling cascade connecting the receptors and flagellar motors, which also allows the cell to adapt to varying levels of external stimuli.

While the physiological characteristics of individual species may vary, for instance, E. coli rely on a series of flagella to propel them through their environment whereas R. sphaeroides cells have only one flagellum, the above described sensory components are similar in many species (see Figure 1 for an illustration of the differences and similarities between three examples).

Most bacteria have receptors for both attractants and repellents, although research has generally favored the addition or removal of attractants. In what follows we will discuss the bacterial response to an attractant gradient unless otherwise indicated. The overall response process can be considered as consisting of the following four well-defined phases. 


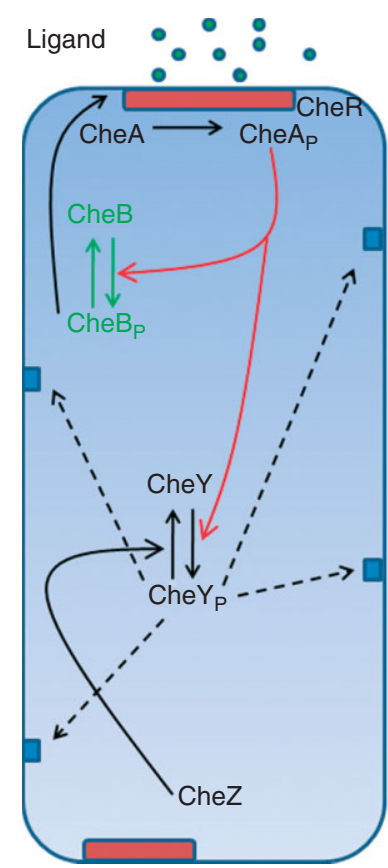

(a)

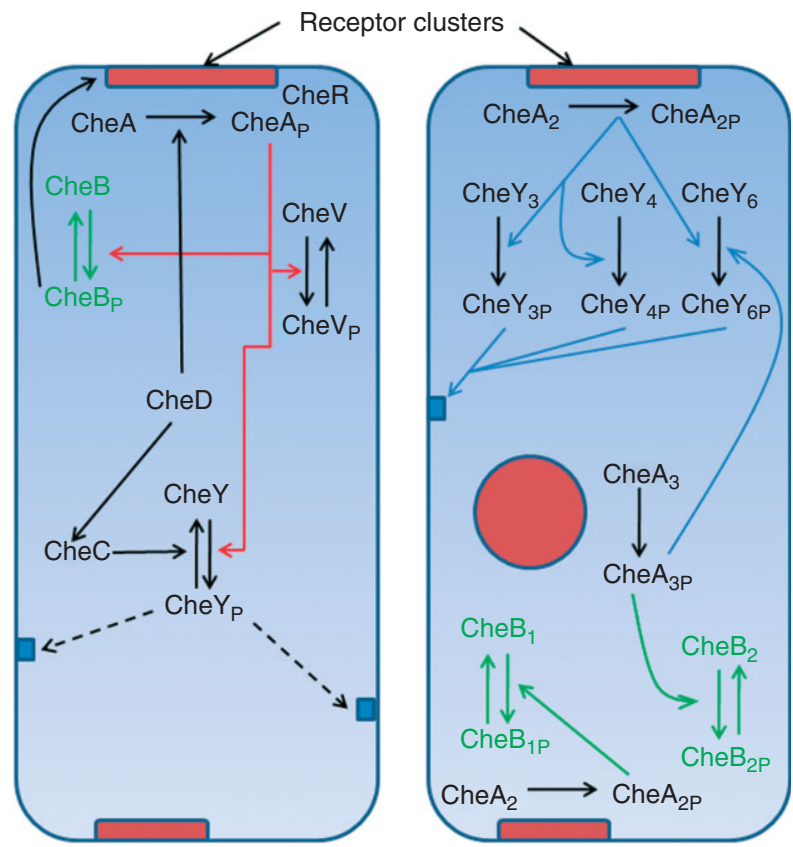

(b)

(c)

FIG URE 1 | A schematic comparison of the intracellular signaling pathways and related components (receptor clusters and flagellar motors) in: (a) E. coli, (b) B. subtilis, and (c) R. sphaeroides chemotactic bacteria. Each cell may detect an external attractant or repellent gradient by ligand molecules binding to a receptor cluster (red boxes). These then signal via the intracellular biochemical cascades using a histidine kinase, CheA, regulating the CheBs which control the adaptation pathways, and CheYs which control the flagellar motor switching (smaller blue boxes on the cell membrane). CheZ is a phosphatase which dephosphorylates phosphorylated CheY. The complexity of each pathway varies considerably. Although $E$. coli and $B$. subtilis each contain one $\mathrm{CheY}$ (the role of $\mathrm{CheV}$ is not fully understood in $B$. subtilis) and multiple flagella, $R$. sphaeroides requires a minimum of three CheYs, of a total of six, for chemotaxis. It also comprises cytoplasmic-based receptor clusters which probably sense intracellular metabolites. The $B$. subtilis cascade is also augmented by two further proteins CheC and CheD. CheC is involved in a second adaptation pathway by binding with CheY-P and CheD. ${ }^{2}$ CheD further acts as a phosphatase for CheY-P. ${ }^{3}$ In each bacterium CheR is the methyltransferase which works with CheB to control adaptation.

1. The sensory MCP receptors detect changes in the concentration of the extracellular ligand attractant via binding of the ligand to them.

2. Sensory detection of an increase in attractant leads to the cessation of autophosphorylation of MCP cytoplasmic bound kinase proteins. This in turn leads to a reduction in the phosphorylation levels of intracellular signaling proteins.

3. The change in phosphorylated flagellar motor signaling proteins, via the signaling cascade, is detected at the motor leading to a switch in rotational direction.

4. The sensory receptors adapt to the originally detected change in step 1 by re-setting the receptors via the process of methylation which is controlled via changes in intracellular signaling proteins specific to adaptation.

The final phase returns the system to its initial pre-stimulus state leaving it free to detect further changes in the extracellular ligand concentration. The time taken for each phase to occur varies considerably; ligand detection is of the order of milliseconds, intracellular signaling to the flagellar motors is of the order of hundreds of milliseconds and adaptation can vary anywhere between tens to hundreds of seconds, dependent on the strength of the stimulus.

This sensory process allows the bacteria to control their flagella and therefore how they move through their environment. For instance, when the approximately 4-6 flagella located in the membrane of an E. coli cell rotate clockwise (CW) they fly apart causing the cell to re-orientate (a tumble) in a new random direction. However, when rotated counter-clockwise (CCW) all of the flagella come together in a single bundle propelling the cell forward in one direction for an extended period of time (a run) as shown in Figure 2. In the absence of an attractant gradient an E. coli cell will utilise a combination of these short runs and tumbles to move randomly through its 


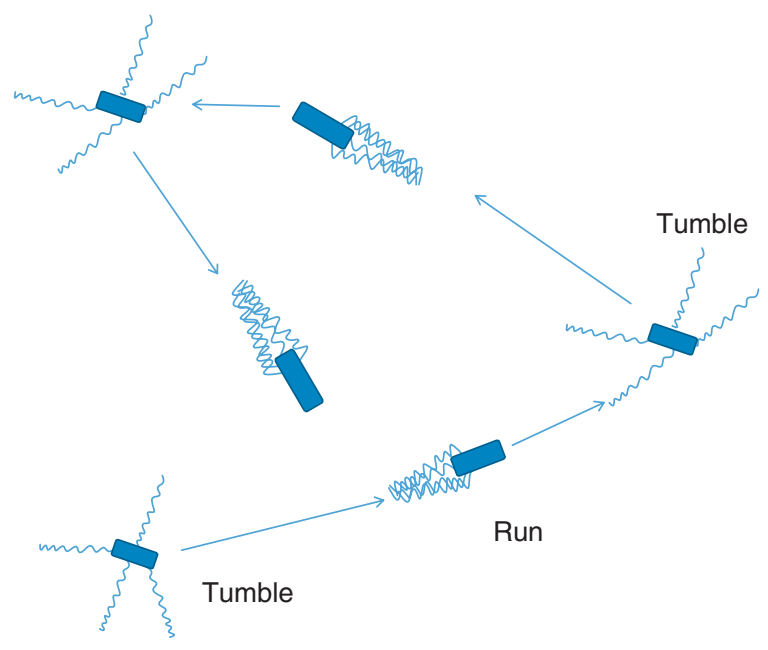

FIGURE 2 | In a uniform environment Escherichia coli alternates between runs, where all the flagella rotate counter-clockwise as a bundle, and tumbles, where some switch to clockwise rotation and the bundle falls apart. When going up an attractant gradient the period between switching increases, when going down a gradient it decreases.

environment. Upon detection of an attractant gradient the duration of the runs is extended, at the expense of a reduction in the number of tumbles, causing net motion in the direction of the attractant gradient. It is this latter response which is commonly referred to as chemotactic cell motion or chemotaxis for short.

Why is bacterial chemotaxis a paradigm for Systems Biology? The field, unlike many other areas of cell or molecular biology, benefits from nearly 40 years of quantifying specific aspects of the signal transduction pathway within E. coli. Until more recent years this has been the chosen bacterium of study for many researchers. For instance, much of the signal transduction kinetics within E. coli has been quantified, concentrations of all of the signaling proteins are known, and details regarding protein diffusion are available, as detailed in Table 1 . With such a wealth of data one would assume that many of the questions regarding bacterial behavior and signaling can be answered without recourse to theoretical modeling. On the contrary, the complexity of the signaling systems, the various temporal and spatial scales over which components of the bacteria (receptors, intracellular signaling cascade, and motor) operate, the interactions both within and between these components plus the experimental restrictions on being able to observe internal processes dynamically in vivo, means theoretical modeling is necessary to overcome experimental limitations and provide new insights and predictions regarding bacterial function and behavior.
TABLE $1 \mid$ An Overview of the Known Protein Kinetic, Concentration, and Diffusion Parameters for the Escherichia coli Chemotaxis Signaling Cascade

\begin{tabular}{|c|c|c|}
\hline Description & Value & References \\
\hline Autophosphorylation of CheA & $34 s^{-1}$ & 4,5 \\
\hline $\begin{array}{l}\text { Phosphotransfer from } \mathrm{CheA}_{P} \\
\text { to CheY }\end{array}$ & $1 \times 10^{8}(\mathrm{Ms})^{-1}$ & 6 \\
\hline $\begin{array}{l}\mathrm{CheY}_{P} \text { dephosphorylation } \\
\text { by CheZ }\end{array}$ & $1.6 \times 10^{6}(\mathrm{Ms})^{-1}$ & 7,8 \\
\hline $\mathrm{CheY}_{p}$ natural dephosphorylation & $8.5 \times 10^{-2} s^{-1}$ & 9,10 \\
\hline $\begin{array}{l}\mathrm{CheY}_{P} \text { intracellular diffusion } \\
\text { coefficient }\end{array}$ & $10 \mu \mathrm{m}^{2} \mathrm{~s}^{-1}$ & 11,12 \\
\hline $\begin{array}{l}\text { Total* average CheA concentration } \\
\text { in a cell. }\end{array}$ & $7.9 \mu \mathrm{M}$ & 7 \\
\hline $\begin{array}{l}\text { Total average CheY concentration } \\
\text { in a cell }\end{array}$ & $9.7 \mu \mathrm{M}$ & 7 \\
\hline $\begin{array}{l}\text { Total average CheB concentration } \\
\text { in a cell }\end{array}$ & $0.28 \mu \mathrm{M}$ & 7 \\
\hline $\begin{array}{l}\text { Total average CheZ concentration } \\
\text { in a cell }\end{array}$ & $3.8 \mu \mathrm{M}$ & 7 \\
\hline
\end{tabular}

The availability of such data makes the system very attractive to theoretical modelers who are able to apply quantitative modeling techniques to further understand specific aspects of the signaling system and the overall bacterial response.

*Sum of total unphosphorylated and phosphorylated protein.

\section{THEORETICAL MODELS OF BACTERIAL CHEMOTAXIS}

A considerable range of theoretical models focusing on different aspects of the bacterial chemotactic system have been developed over the last 40 years. The interested reader may wish to consult the more detailed reviews of Refs 13 and 14 for further details.

On the single cell scale theoretical models have focused on: (1) explaining gain at the receptor clusters upon ligand binding via co-operativity of the receptors; (2) describing various ways in which adaptation can be achieved by specific parts of the bacterial system; (3) modeling the intracellular signaling cascade; (4) elucidating aspects of the interplay between gain, adaptation, and signaling; (5) understanding flagellar motor activation and function; and (6) elucidating the role of cell-fluid dynamical interactions on cell motility, especially at surfaces. The contribution of theoretical modeling to each of these areas is reviewed in the following sections.

Theoretical approaches have ranged from the use of continuous deterministic methods such as ordinary differential equations ${ }^{15-20}$ and partial differential equations ${ }^{21,22}$ to statistical mechanics, ${ }^{23-25}$ discrete stochastic models ${ }^{26-29}$ as well as combined approaches. ${ }^{30}$ In the early days many models on the single cell scale were developed with limited 
experimental data to confirm their findings. The models made theoretical predictions on how the internal machinery of the bacteria might operate to achieve the observed experimental behavior, thus providing impetus for experimental research into particular aspects of the bacterial system.

As time has progressed and experimental knowledge has grown, the results of developed models are now compared with experimental findings to either test the model's ability to reproduce experimental findings, ${ }^{23,30}$ or provide insight into how the system may operate, ${ }^{31}$ without recourse to a large number of experiments. In this sense theoretical modeling and experimental work have progressed hand-in-hand in developing our understanding of bacterial chemotactic species.

Although the vast majority of theoretical models have been developed in the context of E. coli, work in recent years has also begun to focus on examining signaling processes in B. subtilis ${ }^{32}$ and R. sphaeroides. ${ }^{31,33,34}$

\section{RECEPTOR MODELING, GAIN, AND ADAPTATION}

The ability of a bacterium to sense its biochemical environment across five orders of variation in background concentration and initiate the intracellular signaling cascade when it detects a gradient consisting of as little as five ligand molecules, brings together two important aspects of the bacterial system: (1) the ability to detect extracellular changes (and amplify them in the case of quantitatively small changes in ligand concentration) which initiate the signaling cascade (gain); and (2) the ability to return the sensory system to its pre-stimulus configuration (adaptation). While they can be considered in their own right, both aspects are closely interwoven. Theoretical models formulated to answer questions regarding how bacterial cells can achieve gain across varying concentration gradients have incorporated details of receptor signaling and adaptation in an attempt to answer this question.

Work in the 1970s and 1980s in the field of receptor signaling focused on explaining adaptation through simple models of receptor-ligand binding. Ordinary differential equation models were often used to model the respective dynamics and understand how the interactions between the receptor states and ligand binding could lead to exact adaptation (the cell's ability to return exactly to its pre-stimulus levels of the intracellular signaling proteins). ${ }^{16,17,35,36}$ These theoretical models played an important role; little was known about the proteins which regulated the receptor and adaptation system and thus modeling was able to provide insight into known mechanisms and whether they alone could provide the basis for the observed adaptation and gain. In the case of adaptation these approaches were relatively successful, but would later be updated to incorporate further experimental details as they emerged. The ability to identify possible mechanisms for gain remained elusive.

Considerable theoretical work was undertaken in the 1990s by Dennis Bray and colleagues in modeling receptors and their ability to initiate gain. Models of the intracellular signaling network which included simple receptor signaling had been unable to explain gain within the system. ${ }^{37}$ This work focused initially on modeling the formation of receptor clusters, ${ }^{38}$ specifically the receptors and the receptor-related kinase CheA, but later shifted to understanding the role that receptor clustering may play in gain. Following experimental evidence regarding the possibility of receptors operating in clusters, developed a now seminal piece of modeling work to Bray and colleagues ${ }^{39}$ show that clustering rather than individual receptor signaling could greatly influence gain. From this followed a series of receptor-based models utilizing the well-known Ising model to understand the role of clustering. ${ }^{40-44}$

In recent years the focus of theoretical modeling in understanding signaling has shifted to detailed models which include receptors and their ability to cluster (see Figure 3), the role of the adaptation proteins $\mathrm{CheR}$ and $\mathrm{CheB}$ and receptor methylation. Of particular note has been the work of authors who have developed energy-based Monod Wyman Changeux (MWC) models from statistical physics which consider the effect of varying the receptor cluster size on both gain and adaptation. ${ }^{23,24,45}$ Model results are in good agreement with findings and the clustering mechanism

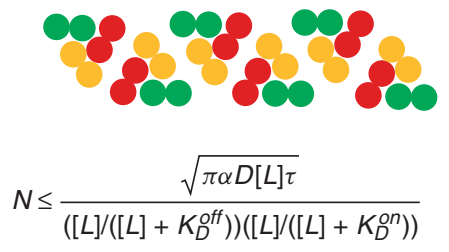

FIG URE 3 | Upper: Trimers of receptor dimers cluster as viewed from above (after Ref 48). The homodimers (indicated by circles of the same color) interact with other dimers to form a cluster of heterotrimers of homodimers. Lower: The statistical mechanically derived inequality ${ }^{49}$ describes the theoretical maximum cluster size $N$ of trimers of dimers as a function of the ligand diffusion rate $D$, the average receptor size $a$, the average diffusion ligand time $\tau$, the ligand concentration $L$, and the receptor-ligand binding affinities $K_{D}^{\text {on }}$ and $K_{D}^{\text {off }}$ (note the superscripts do not denote a mathematical power). Fitting of such relationships to experimental data has allowed predictions to be made as to how cluster size varies amongst receptor types and under different experimental conditions. 
has been able to explain gain over a wide range of background concentrations. In addition, recent work by Shimizu and colleagues ${ }^{46}$ has used FRET data along with a combined ODE-MWC modeling approach ${ }^{47}$ to show the importance of dynamic feedback between the receptor clusters and adaptation signaling process in driving the cellular response to temporally varying attractant gradients. A similar model which captures more details of the intracellular signaling cascade in E. coli and shows good agreement with experimental data has been developed by Endres and colleagues. ${ }^{30}$

\section{SPATIOTEMPORAL PROTEIN LOCALIZATION}

With increased understanding of the spatial colocalization of proteins to specific parts of the cell, ${ }^{50}$ there has been a small, but growing amount of work which has focused on the effect this has on chemosensory activity. The interested reader is directed toward the review in Ref 51 on reaction diffusion systems in intracellular signaling systems for a more detailed discussion on the topic in cell systems.

Lipkow and colleagues showed in 2005 how a spatially explicit model of intracellular signaling proteins could be used to predict the probable location of the phosphatase CheZ within E. coli. ${ }^{28}$ Using a discrete stochastic model of protein diffusion within an E. coli cell, they were able to vary the CheZ concentration throughout the cell to compare the effect of it being evenly distributed between the polar clusters and cytoplasm of the cell to being located at the poles. In respect of the former, simulation results showed that the variation in phosphorylated CheY would be too large within the cell to allow it to respond appropriately to external signaling; given the random location of flagella along the cell surface, disparate levels of phosphorylated CheY would mean some flagella would rotate CW and others CCW and thus the cell would never chemotax. In contrast the localization of CheZ to the polar clusters means dephosphorylation of phosphorylated CheY does not occur along the length of the cell as it diffuses and thus the concentration level is relatively constant along the cell length. This work promoted experimental investigation of CheZ, ${ }^{52}$ confirming the theoretical predictions. Rao and colleagues undertook similar work in a study of how phosphatase localization affects spatial gradients of CheY in E. coli and B. subtilis. ${ }^{21}$ They adopted a continuum approach using a system of reaction diffusion equations to show CheY levels are relatively spatially constant in both bacteria, although the phosphatase is located at the polar clusters in E. coli, but at the flagellar motors in B. subtilis.
More recently Tindall and colleagues ${ }^{22}$ have elucidated how complex formation of signaling proteins in E. coli is important for ensuring appropriate levels of phosphorylated and unphosphorylated $\mathrm{CheY}$ are maintained throughout the cell cytoplasm. By using a continuum partial differential approach they showed how complex formation varies with CheZ content within the cell and how this affects the total amount of free and bound CheY in the cell and at the polar clusters. Their results were verified against the experimental findings of Ref 53.

Relevant to bacterial cell division, a number of authors ${ }^{54-56}$ have focused on the oscillating Min system. Here a number of reaction diffusion type models have been developed to elucidate the importance of reaction and diffusion in defining a spatially oscillating pattern of Min proteins which allows the cell to divide.

\section{MODELING THE BACTERIAL FLAGELLAR MOTOR}

The bacterial flagellar motor is a complex rotary nanomachine, composed of around 20 protein components (none greater than $100 \mathrm{kDa}$ in size), driven by ion flow across the cytoplasmic membrane of a cell and of the order of nanometers in diameter; motors are capable of rotating at hundreds of Hertz enabling the bacterium to propel itself through its extracellular environment. ${ }^{57-59}$ A schematic representation of the E. coli flagellar motor is shown in Figure 4. While much is known about flagellar construction, less is known about their motor function. A number of theoretical approaches have been adopted to help answer questions in the area, in particular the relationship between motor frequency and torque, how torque is generated by the proton motive interaction between the electrostatic proton flow and the motor and the interaction between cytoplasmic motor driving, proteins and the motor. We briefly highlight a few of these areas here. The reader is directed toward the reviews of Refs 60 and 61 for more detailed overviews of theoretical modeling work undertaken in this area.

Mathematical models have generally considered how ion flow through the motor or motor driving protein binding affects function. Examples of the former include a study by Meister and colleagues ${ }^{62}$ who used physical arguments to derive relationships between the motor turning frequency and the flow of ions through the motor and showed that the flagellar rotation (at zero load) was limited by the association and disassociation of the proton ions. Elston and Oster $^{63}$ used a Markov chain model 
(a)

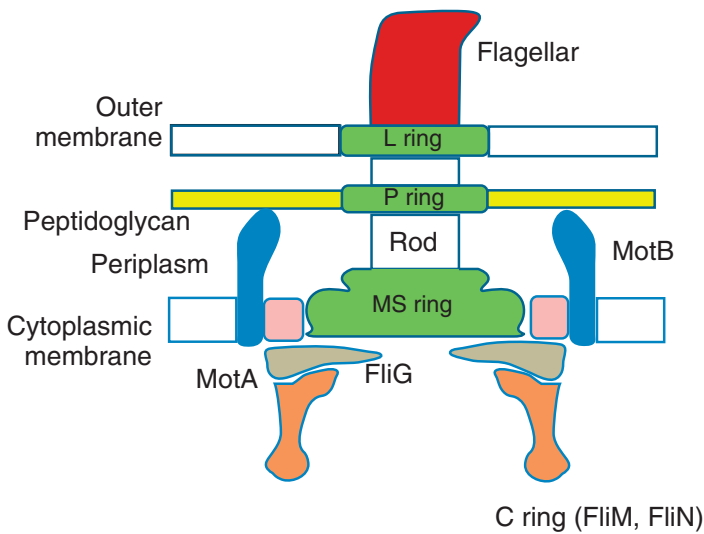

(b)
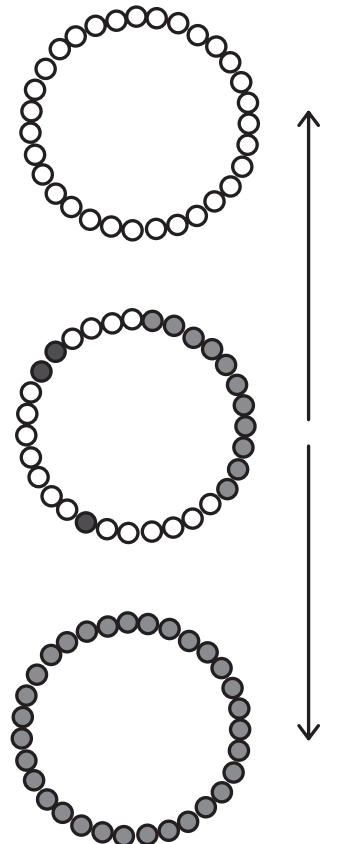

FIGURE 4 | (a) The components of an E. coli bacterial motor. (b) The concept of conformational spread. In the upper figure none of the 32 proteins in the C-ring of the bacteria are activated. Over time, some of the proteins may become activated (gray-colored circles) through stochastic fluctuations or signaling. The theorized connectivity amongst the proteins leads to a spread of this activity (middle figure) which can either increase (lower figure) or decrease, back to the original state (upper figure).

of proton occupancy of the motor, modeled using differential equations, to develop an electromechanical model. The transition rates between the proton occupancy states were dependent upon the proton diffusion and angular position of the rotor, the latter described by the solution of a stochastic Langevin equation incorporating the local effects of motor load, load torque on the rotor, and any associated Brownian noise from interactions with the extracellular environment. Parameterized with experimental data from the literature, this detailed model was able to reproduce many experimental findings and showed that the number, depth, and spacing of the proton potential wells as well as the stator's dimensions and dielectric constants are critical in determining the size of the torque generated. Most recently Xing et al. ${ }^{64}$ have shown how a similar approach can be used to derive a single Markov-Fokker-Planck dynamic equation which accounts for the motor rotor load and orientation. They utilize the model to elucidate certain features of the torque-frequency curve, in particular its rapid decrease at high rotational rates and the relationship between stator number and torque at high frequency.

In the case of interactions between flagellar motors and their cytoplasmic switch driving protein CheY, a discrete stochastic model has been developed to understand how the random binding of CheY-P proteins to the motor-binding protein FliM leads to the motor switching from either a CCW rotation to $\mathrm{CW}$ or vice-versa. ${ }^{65}$ The authors used a Monte Carlo approach to show that once a critical number of FliM-binding sites are occupied by CheY-P the conformational stator ring is able to flip from one state direction to the other dependent upon the assumed coupling strength between the FliM proteins in the ring (see Figure 4(b)). The concept of this form of co-operativity within the flagellar motor has recently been tested experimentally, ${ }^{66}$ with good agreement between theory and experiment.

\section{EVOLUTION IN BACTERIA}

How bacteria have evolved to exhibit such complex behavior as the run-and-tumble process, including their internal machinery which facilitates this, has been the subject of a recent growing area of research in bacterial chemotaxis. As noted by Soyer, ${ }^{67}$ two approaches are being taken. The first is the analysis of genomic sequences to understand the relationship between various bacterial species in terms of their phylogeny. Such bioinformatic approaches have identified a common core of chemosensory proteins, usually encoded in ordered operons, across most motile species, and also suggested evolutionary routes for the development of motors from transport systems. 
The second approach involves developing simulation models of bacteria, which, under certain rules of evolution, acquire various characteristics, such as the run-and-tumble process. By altering the rules, one is able to observe their effect on 'evolution' thus allowing mechanisms to be inferred. One such example is the work of Nicolau and colleagues (Personal communication) who showed that the run-and-tumble process can be obtained by a set of simple optimization rules. Similar approaches have been adopted by other authors and the reader here is directed to the brief review in Ref 67 for further details.

\section{INTERACTIONS WITH THE EXTRACELLULAR PHYSICAL ENVIRONMENT}

Classical mechanisms for prokaryotic chemotaxis are governed by the interaction between intracellular signaling and the regulation of external rotating flagella for a swimming cell, which immediately couples the chemotactic system to the surrounding physical environment. For an individual bacterium far from surfaces, the latter couplings are not particularly important. However, flagellated bacteria may strongly influence the trajectories of nearby cells ${ }^{68}$ and, in dense populations, induce extensive collective effects, ${ }^{69,70}$ capable of altering the chemotactic gradient. Furthermore, for cell swimming via a single flagellum, or flagellar bundle, the presence of a surface will typically induce curved swimming trajectories simply by hydrodynamical interactions, ${ }^{71-73}$ again altering the chemotactic response. Hence, the feedback between hydrodynamics and chemotaxis is especially important in numerous contexts, such as population behavior and biofilm seeding, in turn motivating extensive modeling of bacterial swimming fluid dynamics.

There is an extensive array of techniques used for the study of microbial motility fluid dynamics, as surveyed in Refs 74 and 75 . The easiest to implement, but the least flexible and accurate, is resistive force theory, as first used by Gray and Hancock, ${ }^{76}$ and applied to helical flagella in numerous studies, for instance Refs 77, 78, and 72. This approach gives a relationship between the velocity and angular velocity on the one hand and the viscous drag force and torque on the other for the flagellum, though only to logarithmic accuracy in the flagellum slenderness and with the entirely legitimate assumption that inertial effects are negligible. In conjunction with analogous relationships for the cell body, one can predict, for example, numerous kinematic relations, such as swimming trajectories in terms of flagella movements, which have been validated using observations of swimming eukaryotes (See Ref 79 for example).

In particular, for monotrichous bacteria or cells with flagellar bundles, resistive force theory can relate total motor torques to swimming velocities. These calculations can be readily extracted from the work by Lauga and colleagues ${ }^{72}$ given a spherical cell body; this study also includes approximations accounting for the influence of a nearby surface, thus highlighting the extensive increase in complexity for resistive force theory calculations in the presence of a surface. Furthermore, resistive force theory is not applicable for swimmers in close proximity and more generally the limitations in its applicability are highlighted in detailed numerical studies. ${ }^{80,81}$

Hence, more accurate and general methodologies have been developed. A fast and easily implemented numerical method captures the flagellum dynamics via slender body theory (e.g., Refs 81,82), often in combination with boundary element methods for the cell body mechanics (e.g., Ref 83). While a significant improvement is observed on resistive force theory, slender body theory is still an approximation and high flagellar rotation rates significantly reduce its accuracy for prokaryotes compared to eukaryotic flagellar beating. ${ }^{84}$ However, an array of direct numerical simulation techniques are also available with no such limitations, as briefly reviewed in Ref 85. In particular regularized Stokeslet, immersed boundary and boundary element methods have been implemented for bacteria, though such techniques are more time consuming and computationally intensive to implement. ${ }^{68,86-92}$

These studies confirm that nearby bacteria influence each other's trajectories simply by hydrodynamics ${ }^{68,91}$ demonstrating that physical interactions are important, and also complex, for swimming bacteria. In addition, detailed simulations of bacterial behavior near surfaces highlight a sensitivity to cell geometry to the extent that significant variations would be expected within single species populations of cells. Indeed, changes in the morphology of the E. coli cell body, resulting in different surface swimming trajectories, have been exploited for fractionation according to cell age. ${ }^{93}$ Such subtleties emphasize the complexities in developing a modeling framework for understanding and predicting geometrically constrained chemotactic behaviors, as swimming trajectories are fundamentally altered by surface interactions. A further difficulty arises in scaling individual behaviors to the population level, while incorporating fluid dynamical effects; in particular although simulations exploring very small numbers of geometrically accurate swimmers have been considered, 
these models cannot be scaled to population levels. Nonetheless, the fluid dynamics associated with large numbers of idealized swimmers has been investigated, typically modeling cells as dipoles of point forces, representing a net flagellar propulsion force coupled with a concomitant cell body drag force (e.g., Refs 94,95). Simulations of chemotactic cells have also been recently considered in this manner, studying when homogeneous cell suspensions destabilize. ${ }^{96}$

However, whether the use of point force dipole models can accurately assess cell dynamics remains to be considered in detail and is unexplored in the context of how the flagellated motility of dense bacterial populations influences the velocity field. In contrast, the subtleties observed in geometrically accurate simulations of surface swimmers ${ }^{92}$ strongly indicate that idealized swimmers models near surfaces or in confined regions are likely to require more refined representations than dipolar forces within modeling frameworks. Incorporating such features within population models is already recognized as a fundamental area for multiscale modeling research ${ }^{97}$ and critical for understanding the dynamical interplay of chemotaxis and hydrodynamics at the population level.

\section{FROM THE SINGLE CELL TO POPULATION SCALE}

The interaction of flagellated hydrodynamics and chemotaxis is one example of the fact that whilst understanding bacteria at the single cell level is necessary for an elucidation of how large numbers of cells will behave, it is not sufficient. The 'linking' of single cell to population behavior is an important challenge

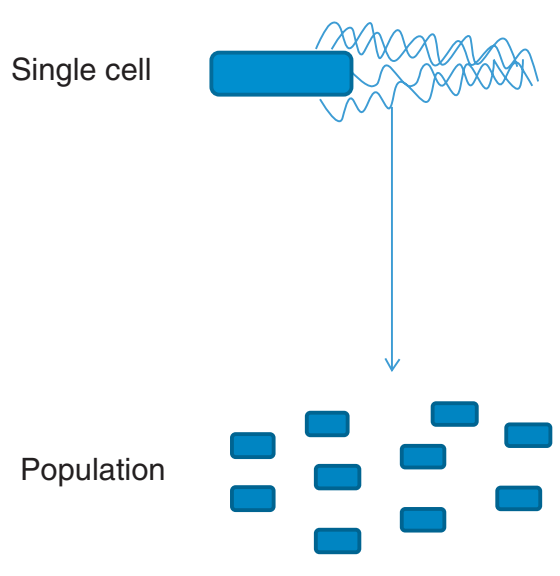

across many areas of Systems Biology research and one where progress is required for insight gained at the single cell level to be applied in understanding real-world problems at the higher population scale (see Figure 5).

In the context of classical bacterial chemotaxis studies, efforts have been ongoing for over 30 years to link the two scales. The main focus of such work, initially, was how macroscopically measured variables such as diffusion rates could be linked to measurable individual cell properties, such as cell speed and turning angle. In 1977, Segel linked the diffusion and chemotactic coefficients of a spatiotemporal model of a bacterial chemotactic population (the now wellknown Keller-Segel formulation) with microscopic variables describing the average cell velocity, the rates at which the bacteria moved between states describing their spatial position, and receptor-ligand occupancy. Such work has led to a steady number of publications since that have attempted to link microscopic (individual) and macroscopic (population) behavior. ${ }^{98}$ Of particular note is the study by $\mathrm{Alt}^{99}$ who incorporated details of the cell turning angle and mean speed into a stochastic description, and the subsequent work by Rivero and colleagues ${ }^{100}$ who looked at the connection between cell speed, run persistence time, and ligand-receptor occupancy. Considerable comparisons and analysis of these and associated models have been undertaken in Refs 101-105.

More recent work includes that of Refs 106 and 107, 108. The former formulated and used simple descriptions of excitation and adaptation in a Monte Carlo simulation of a bacterial population to show how coarse-graining of the resultant population behavior could be used to decrease computational time

\section{Intracellular signaling cascade}

Flagellar motors

Receptor signaling and gain

Hydrodynamics

Discrete deterministic and stochastic simulations

Continuum descriptions (Partial differential equations).

Modeling environmental conditions (nutrient, fluid quorum sensing, etc.)

FIG URE 5 | A summary of the main components of bacterial chemotactic species which have been modeled on the single cell and population scale. For an understanding of how individual-based processes (e.g., intracellular signaling cascades) affect the overall population scale behavior links between the two scales are required. 
in determining the population behavior. The latter utilized analytical techniques to move from the individual cell description of excitation and adaptation formulated in Ref 106 to a partial differential equation description of a bacterial population's spatiotemporal dynamics. de Gennes ${ }^{109}$ and Clark and Grant ${ }^{110}$ have considered how response functions reflecting the runand-tumble process on the single cell scale can lead to variation in population behavior in the presence of a chemoattractant. In particular Clark and Grant demonstrated that optimizing the individual bacterial response does not lead to optimized population aggregation at high chemoattractant concentrations. More recently Celani and Vergassola ${ }^{111}$ have shown how bacterial chemotaxis is a maxmin strategy in complex multiple stimuli environments; chemotactic bacteria will always try to maximize their response to even low minimal chemoattractant concentrations. Their work utilizes model simulations and experimentation and results are discussed in the context of game theory and evolution.

Bray and colleagues have developed a number of computational models which allow single cell properties, for instance details of the signaling cascade, to be incorporated into models and thus enable us to understand the effect that variations in these processes have on the population behavior. The E. solo model from Bray and colleagues ${ }^{112}$ includes a description of the intracellular signaling cascade with each individual bacterium having up to four flagella in a two spatial dimension attractant gradient. The in silico bacteria are subjected to uniform attractant gradients of aspartate which can be adjusted during the simulations and measurements of the tumbling frequency, turn angle, and adaptation time of the population can be recorded. The analysis of pre-defined fixed attractant gradients for mutant and wild-type bacteria without flagella was also considered in the paper; this model referred to as E. pluribus. In particular, these studies were used to show the effect of adaptation and individual cell susceptibility to attractant binding on bacterial aggregation, for instance, how cells do not necessarily accumulate in regions of highest attractant concentration because of adaptation.

Of further relevance to this area is the mathematical modeling work which has focused on reproducing many of the patterns observed in the culturing of bacterial cells in the laboratory. ${ }^{113-116}$ Such work has often used the well-known Keller-Segel model ${ }^{117}$ to understand how bacterial cell and nutrient diffusion and the interactions between them drive pattern formation in bacterial cell cultures. ${ }^{118-120}$ Further discussion on this work is outside the scope of this review, but the interested reader should consult the review provided in Ref 108 for further information.

\section{SUMMARY AND CONCLUSIONS}

The combined efforts of theoretical modelers and experimentalists in advancing understanding of the bacterial chemotaxis system, means the field is an excellent example of Systems Biology research 'in action'. The regular publication of articles where combined theoretical and experimental work has led to new insights or the publication of purely theoretical or experimental work, where one has informed the other, have created a benchmark for other areas of cell biology. If the iterative process of theoretical model development, experimental testing, and the use of theoretical predictions in informing the particular system, or problem being studied, can be applied in other biological contexts at the single and multicell level, then Systems Biology will be sure to make contributions to important scientific questions in the short and longer term.

The field itself is developing in a range of areas as this review has highlighted. Given much that is now known in the context of E. coli chemotaxis, although there is still much more to be understood, there has been a growing trend in recent years for developing similar understanding in other bacterial chemotactic species. Again such work is backed by a number of years of quantitative experimentation which the field of bacterial chemotaxis is able to provide. Thus, the field is ripe for the continued development of new theoretical models, such as those outlined in this review, but is also positioned to take advantage of advances in molecular and cellular biology. For instance the recent development of techniques for tracking single molecules within cells ${ }^{121}$ will provide new challenges for theoreticians in modeling single cell phenomena in crowded cell environments.

Recent theoretical work also includes the elucidation of possible signaling cascades within R. sphaeroides by Hamadeh and colleagues ${ }^{33}$ who have focused on theoretically inferring the form of intracellular signaling networks from flagellar rotational data. The theoretical networks can then be tested experimentally to either validate or invalidate them. The ability of bacterial cells to respond to the same multiplicative variation in attractant concentration (e.g., $0.1-0.01 \mathrm{mM}$ and $1-10 \mathrm{mM}$ both represent a 10 -fold change in concentration) without any variation in their internal response was theoretically predicted by Shoval and colleagues ${ }^{122}$ in 2010 and recently shown experimentally in Ref 123.

One of the great challenges for not only the field of bacterial chemotaxis modeling, but that of 
the application of theoretical models to many areas of Systems Biology (and indeed associated areas such as physiology), is overcoming computational time in linking models of lower cellular length scale behavior with that of the tissue or in the case of bacteria and biofilm scale. To provide in silico predictions on the many cell $\left(\sim 10^{8}\right)$ biofilm scale is extremely costly in terms of time for many computational models which have included detail of the cellular signaling processes. The increase in further computing power will go some way in helping with this problem, but theoreticians must also consider ways in which links, both spatially and temporally, can be made between the discrete cellular and continuum many cell scales if progress in applying knowledge at the single cell scale to real-world problems is to be made.

\section{ACKNOWLEDGMENTS}

M.J.T. was supported by a Research Council UK Fellowship during the period in which this work was undertaken. P.K.M. was partially supported by a Royal Society Wolfson Research Merit Award. Research in the J.P.A. laboratory is supported by funding from the Biotechnology and Biological Sciences Research Council.

\section{REFERENCES}

1. Eisenbach M, Lengeler JW, Varon M, Gutnick D, Meili R, Firtel RA, Segall JE, Omann GM, Tamada A, Murakami F. Chemotaxis. London: Imperial College Press; 2004.

2. Rao CV, Glekas GD, Ordal GW. The three adaptation systems of Bacillus subtilis chemotaxis. Trends Microbiol 2008, 16:480-487.

3. Porter SL, Wadhams GH, Armitage JP. Signalling processing in complex chemotaxis pathways. Nat Rev Microbiol 2011, 9:153-165.

4. Francis NR, Levit M, Shaikh TR, Melanson LA, Stock JB. Subunit organization in a soluble complex of Tar, CheW and CheA by electron microscopy. J Biol Chem 2002, 277:36755-36759.

5. Shrout AL, Montefusco DJ, Weis RM. Templatedirected assembly of receptor signalling complexes. Biochemistry 2003, 42:13379-13385.

6. Stewart RC, Jahreis K, Parkinson JS. Rapid phosphotransfer to CheY from a CheA protein lacking the CheY-binding domain. Biochemistry 2000, 39:13157-13165.

7. Li M, Hazelbauer GL. Cellular stoichiometry of the components of the chemotaxis signalling complex. J Bacteriol 2004, 186:3687-3694.

8. Sourjik V, Berg HC. Binding of the Escherichia coli response regulator CheY to its target measured in vivo by fluorescence resonance energy transfer. Proc Nat Acad Sci 2002, 99:12669-12674.

9. Smith JG, Latiolais JA, Guanga GP, Citineni S, Silversmith RE, Bourret RB. Investigation of the role of electrostatic charge in activation of the Escherichia coli response regulator CheY. J Bacteriol 2003, 185:6385-6391.

10. Stewart RC, van Bruggen R. Rapid phosphotransfer to CheY from a CheA protein lacking the CheY-binding domain. Biochemistry 2004, 43:8766-8777.
11. Elowitz MB, Surette MG, Wolf P, Stock JB, Leibler S. Protein mobility in the cytoplasm of Escherichia coli. J Bacteriol 1999, 181:197-203.

12. Segall JE, Ishihara A, Berg HC. Chemotactic signalling in filamentous cells of Escherichia coli. J Bacteriol 1985, 161:51-59.

13. Liebal UW, Millat T, de Jong IG, Kuipers OP, Völker U, Wolkenhauer O. How mathematical modelling elucidates signalling in Bacillus subtilis. Mol Microbiol 2010, 77:1083-1095.

14. Tindall MJ, Porter SL, Maini PK, Gaglia G, Armitage JP. Overview of mathematical approaches used to model bacterial chemotaxis I: the single cell. Bull Math Biol 2008, 70:1525-1569.

15. Block SM, Segall JE, Berg HC. Adaptation kinetics in bacterial chemotaxis. J Bacteriol 1983, 154:312-323.

16. Goldbeter A, Koshland DE. Simple molecular model for sensing and adaptation based on receptor modification with application to bacterial chemotaxis. J Math Biol 1982, 161:395-416.

17. Asakura S, Honda H. Two-state model for bacterial chemoreceptor proteins: the role of multiple methylation. J Math Biol 1984, 176:349-367.

18. Hauri DC, Ross J. A model of excitation and adaptation in bacterial chemotaxis. Biophys J 1995, 68:708-722.

19. Spiro PA, Parkinson JS, Othmer HG. A model of excitation and adaptation in bacterial chemotaxis. Proc Nat Acad Sci 1997, 94:7263-7268.

20. Barkai N, Leibler S. Robustness in simple biochemical networks. Nature 1997, 387:913-917.

21. Rao CV, Kirby JR, Arkin AP. Phosphatase localization in bacterial chemotaxis: divergent mechanisms, convergent principles. Phys Biol 2005, 2:148-158.

22. Tindall MJ, Porter SL, Wadhams GH, Maini PK, Armitage JP. Spatiotemporal modelling of CheY complexes in Escherichia coli chemotaxis. Prog Biophys Mol Biol 2009, 100:40-46. 
23. Endres RG, Wingreen NS. Precise adaptation in bacterial chemotaxis through "assistance neighbourhoods". Proc Nat Acad Sci 2006, 103:13040-13044.

24. Hansen CH, Endres RG, Wingreen NS. Chemotaxis in Escherichia coli: a molecular model for robust precise adaptation. PLoS Comput Biol 2008, 4:e1.

25. Hansen CH, Sourjik V, Wingreen NS. A dynamicsignaling-team model for chemotaxis receptors in Escherichia coli. Proc Nat Acad Sci 2010, 107:17170-17175.

26. Bray D, Duke T. Conformational spread: the propagation of allosteric states in large multiprotein complexes. Annu Rev Biophys Biomol Struct 2004, $33: 53-73$

27. Goldman J, Andrews S, Bray D. Size and composition of membrane protein clusters predicted by Monte Carlo analysis. Eur Biophys J 2004, 33:506-512.

28. Lipkow K, Andrews SS, Bray D. Simulated diffusion of phosphorylated CheY through the cytoplasm of Escherichia coli. J Bacteriol 2005, 187:45-53.

29. Lipkow K. Changing cellular location of CheZ predicted by molecular simulations. PLoS Comput Biol 2006, 2:301-310.

30. Clausznitzer D, Oleksiuk O, Lłvdok L, Sourjik V, Endres RG. Chemotactic response and adaptation dynamics in Escherichia coli. PLoS Comput Biol 2010, 6:e1000784.

31. Tindall MJ, Porter SL, Maini PK, Armitage JP. Modeling chemotaxis reveals the role of reversed phosphotransfer and a bi-functional kinase-phosphatase. PLoS Comput Biol 2010, 6:e100896.

32. Rao CV, Kirby JR, Arkin AP. Design and diversity in bacterial chemotaxis: a comparative study in Escherichia coli and Bacillus subtilis. PLoS Biol 2004, 2:239-252.

33. Hamadeh A, Roberts MA, August E, McSharry PE, Maini PK, Armitage JP, Papachristodoulou A. Feedback control architecture and the bacterial chemotaxis network. PLoS Comput Biol 2011, 7:e1001130.

34. Roberts MA, August E, Hamadeh A, Maini PK, McSharry PE, Armitage JP, Papachristodoulou A. A model invalidation-based approach for elucidating biological signalling pathways, applied to the chemotaxis pathway in R. sphaeroides. BMC Syst Biol 2009, 31:105.

35. Segel LA. Incorporation of receptor kinetics into a model for bacterial chemotaxis. J Theor Biol 1976, $57: 23-42$

36. Block SM, Segall JE, Berg HC. Impulse response in bacterial chemotaxis. Cell 1982, 31:215-226.

37. Bray D, Bourret RB, Simon MI. Computer simulation of the phosphorylation cascade controlling bacterial chemotaxis. Mol Biol Cell 1993, 4:469-482.

38. Bray D, Bourret RB. Computer analysis of the binding reactions leading to a transmembrane receptor-linked multiprotein complex involved in bacterial chemotaxis. Mol Biol Cell 1995, 6:1367-1380.

39. Bray D, Levin MD, Morton-Firth CJ. Receptor clustering as a cellular mechanism to control sensitivity. Nature 1998, 393:85-88.

40. Shi Y, Duke T. Cooperative model of bacteria sensing. Phys Rev E 1998, 58:6399-6406.

41. Duke TAJ, Bray D. Heightened sensitivity of a lattice of membrane receptors. Proc Nat Acad Sci 1999, 96:10104-10108.

42. Shi Y. Adaptive Ising model and bacterial chemotactic receptor network. Eur Lett 2000, 50:113-119.

43. Shi Y. Effects of thermal fluctuation and the receptorreceptor interaction in bacterial chemotactic signalling and adaptation. Phys Rev E 2001, 64:1-8.

44. Shi Y. Clustering and signalling of cell receptors. Physica A 2002, 311:199-212.

45. Skoge ML, Endres RG, Wingreen NS. Receptorreceptor coupling in bacterial chemotaxis: evidence for strongly coupled receptors. Biophys J 2006, 90:4317-4326.

46. Shimizu TS, Tu Y, Berg HC. A modular gradientsensing network for chemotaxis in Escherichia coli revealed by responses to time-varying stimuli. Mol Syst Biol 6:382-. doi:10.1039/msb.2010.37.

47. Tu Y, Shimizu TS, Berg HC. Modeling the chemotactic response of Escherichia coli to time-varying stimuli. Proc Nat Acad Sci USA 2008, 105:14855-14860.

48. Falke JJ. Cooperativity between bacterial chemotaxis receptors. Proc Nat Acad Sci 2002, 99: 6530-6532.

49. Endres RG, Oleksiuk O, Hansen CH, Meir Y, Sourjik V, Wingreen NS. Variable sizes of Escherichia coli chemoreceptor signalling teams. Mol Syst Biol 2008, $4: 211$.

50. Sourjik V, Armitage JP. Spatial organization in bacterial chemotaxis. EMBO J 2010, 29:2724-2733.

51. Soh S, Byrska M, Kandere-Grzybowska K, Grzybowski BA. Reaction-diffusion systems in intracellular molecular transport and control. Angew Chem Int Ed Engl 2010, 49:4170-4198.

52. Kentner D, Sourjik V. Dynamic map of protein interactions in the Escherichia coli chemotaxis pathway. Mol Syst Biol 2009, 5. doi:10.1038/msb.2008.77.

53. Vaknin A, Berg HC. Single-cell FRET imaging of phosphatase activity in the Escherichia coli chemotaxis system. Proc Nat Acad Sci 2004, 101:17072-17077.

54. Howard M, Rutenberg AD, de Vet S. Dynamic compartmentalization of bacteria: accurate division in E. coli. Phys Rev Lett 2001, 87:2781021-2781024.

55. Meinhardt $\mathrm{H}$, de Boer PAJ. Pattern formation in Escherichia coli: a model for the pole-to-pole oscillations of Min proteins and the localization of the division site. Proc Nat Acad Sci 2001, 98:14202-14207. 
56. Howard M, Rutenberg AD. Pattern formation inside bacteria: fluctuations due to the low copy number of proteins. Phys Rev Lett 2003, 90:1281021-1281024.

57. Berry RM, Armitage JP. The bacterial flagellar motor. Adv Microbial Physiol 1999, 41:291-337.

58. Berg HC. The rotary motor of bacterial flagella. Annu Rev Biochem 2003, 72:19-54.

59. Chen S, Beeby M, Murphy GE, Leadbetter JR, Hendrixson DR, Briegel A, Li Z, Shi J, Tocheva EI, Müller A, et al. Structural diversity of bacterial flagellar motors. EMBO J 2011. doi:10.1038.

60. Berry RM. Theories of rotary motors. Phil Trans $R$ Soc Lond B 2000, 355:503-509.

61. Sowa Y, Berry RM. Bacterial flagellar motor. Q Rev Biophys 2008, 41:103-132.

62. Meister M, Caplan SR, Berg HC. Dynamics of a tightly coupled mechanism for flagellar rotation. Biophys $J$ 1989, 55:905-914.

63. Elston TC, Oster G. Protein turbines. I: the bacterial flagellar motor. Biophys J 1997, 73:703-721.

64. Xing J, Bai F, Berry R, Oster G. Torque-speed relationship of the bacterial flagellar motor. Proc Natl Acad Sci U S A 2006, 103:1260-1265.

65. Duke TAJ, Le Novére N, Bray D. Conformational spread in a ring of proteins: a stochastic approach to allostery. J Mol Biol 2001, 308:541-553.

66. Bai F, Branch RW, Nicolau DV Jr, Pilizota T, Steel BC, Maini PK, Berry RM. Conformational spread as a mechanism for cooperativity in the bacterial flagellar switch. Science 2010, 327:685-689.

67. Soyer O. The promise of evolutionary systems biology: Lessons from bacterial chemotaxis. Sci Signal 2010, 3:pe23.

68. Ishikawa T, Hoto M. Interaction of two swimming Paramecia. J Expt Biol 2006, 209:4452-4463.

69. Dombrowski C, Cisneros L, Chatkaew S, Goldstein RE, Kessler JO. Self-concentration and large-scale coherence in bacterial dynamics. Phys Rev Lett 2004, 93:098103.

70. Sokolov A, Goldstein RE, Feldchtein FI, Aranson IS. Enhanced mixing and spatial instability in concentrated bacterial suspensions. Phys Rev E 2009, 80:031903.

71. Magariyama Y, Ichiba M, Nakata K, Baba K, Ohtani T, Kudo S, Goto T. Difference in bacterial motion between forward and backward swimming caused by the wall effect. Biophys J 2005, 88:3648-3658.

72. Lauga E, DiLuzio WR, Whitesides GM, Stone HA. Swimming in circles: motion of bacteria near solid boundaries. Biophys J 2006, 90:400-412.

73. Berke AP, Turner L, Berg HC, Lauga E. Hydrodynamic attraction of swimming microorganisms by surfaces. Phys Rev Lett 2008, 101:038102.

74. Lauga E, Powers TR. The hydrodynamics of swimming microorganisms. Rep Prog Phys 2009, 72:096601.
75. Gaffney EA, GadhCEla H, Smith DJ, Blake JR, Kirkman-Brown JC. Mammalian sperm motility: observation and theory. Ann Rev Fluid Mech 2011, 43:501-528.

76. Gray J, Hancock GJ. The propulsion of sea urchin spermatozoa. J Exp Biol 1955, 32:802-814.

77. Chwang AT, Wu TY. A note on the helical movement of microorganisms. Proc R Soc Lond B 1971, 178:327-346.

78. Lighthill J. Flagellar Hydrodynamics - JV Neumann Lecture. SIAM Rev 1976, 18:161-230.

79. Friedrich BM, Riedel-Kruse IH, Riedel-Kruse J, Julicher F. High-precision tracking of sperm swimming fine structure provides strong test of resistive force theory. J Exp Biol 2010, 213:1226-1234.

80. Johnson RE, Brokaw CJ. Flagellar hydrodynamics: a comparison between resistive-force theory and slender-body theory. Biophys J 1979, 25:113-127.

81. Spagnolie SE, Lauga E. Comparative hydrodynamics of bacterial polymorphism. Phys Rev Lett 2011, 106:058103.

82. Higdon JJL. Hydrodynamics of flagellar propulsion helical waves. J Fluid Mech 1979, 94:331-351.

83. Smith DJ, Gaffney EA, Blake JR, Kirkman-Brown JC. Human sperm accumulation near surfaces: a simulation study. J Fluid Mech 2009, 621:289-320.

84. Johnson RE. An improved slender-body theory for stokes-flow. J Fluid Mech 1980, 99:411-431.

85. Shum H, Gaffney EA. In: Kim MJ, Steager E, Agung Julius A, eds. Mathematical Models for Individual Swimming Bacteria in Microbiorobotics: Biologically Inspired Microscale Robotic Systems. Elsevier; 2012. ISBN: 9781455778911.

86. Cortez R, Fauci L, Medovikov A. The method of regularized Stokeslets in three dimensions: analysis, validation, and application to helical swimming. Phys Fluids 2005, 17:031504.

87. Hsu C, Dillon RH. A 3D motile rod-shaped monotrichous bacterial model. Bull Math Biol 2009, 71:1228-1263.

88. Phan-Thien N, Tran-Cong T, Ramia M. A boundaryelement analysis of flagellar propulsion. J Fluid Mech 1987, 184:533-549.

89. Ramia M, Tullock DL, Phan-Thien N. The role of hydrodynamic interaction in the locomotion of microorganisms. Biophys J 1993, 65:755-778.

90. Goto T, Nakata K, Baba K, Nishimura M, Magariyama $\mathrm{Y}$. A fluid-dynamic interpretation of the asymmetric motion of singly flagellated bacteria swimming close to a boundary. Biophys J 2005, 89:3771-3779.

91. Ishikawa T, Sekiya G, Imai Y, Yamaguchi T. Hydrodynamic interactions between two swimming bacteria. Biophys J 2007, 93:2217-2225. 
92. Shum H, Gaffney EA, Smith DJ. Modelling bacterial behaviour close to a no-slip plane boundary: the influence of bacterial geometry. Proc R Soc Lond A 2010, 466:1725-1748.

93. Hulme SE, DiLuzio WR, Shevkoplyas SS, Turner L, Mayer M, Berg HC, Whitesides GM. Using ratchets and sorters to fractionate motile cells of Escherichia coli by length. Lab Chip 2008, 8:1888-1895.

94. Saintillan D, Shelley MJ. Instabilities and pattern formation in active particle suspensions: kinetic theory and continuum simulations. Phys Rev Lett 2008, 100:178103.

95. Saintillan D, Shelley MJ. Instabilities, pattern formation, and mixing in active suspensions. Phys Fluids 2008, 20:123304.

96. Subramanian G, Koch DL, Fitzgibbon SR. The stability of a homogeneous suspension of chemotactic bacteria. Phys Fluids 2011, 23:041901.

97. Xue C, Othmer HG, Erban R. From individual to collective behavior of unicellular organisms: recent results and open problems in multiscale phenomena in biology. AIP Conf Proc 2009, 1167:3-4.

98. Tindall MJ, Maini PK, Porter SL, Armitage JP. Overview of mathematical approaches used to model bacterial chemotaxis II: bacterial populations. Bull Math Biol 2008, 70:1570-607.

99. Alt W. Biased random walk models for chemotaxis and related diffusion approximations. J Math Biol 1980, 9:147-177.

100. Rivero MA, Tranquillo RT, Buettner HM, Lauffenburger DA. Transport models for chemotactic cell populations based on individual cell behaviour. Chem Eng Sci 1989, 44:2881-2897.

101. Ford RM, Cummings PT. On the relationship between cell balance equations for chemotaxis cell populations. SIAM J Appl Math 1992, 52:1426-1441.

102. Brosilow BJ, Ford RM, Sarman S, Cummings PT. Numerical solution of transport equations for bacterial chemotaxis: Effect of discretization of directional motion. SIAM J Appl Math 1996, 56:1639-1663.

103. Chen KC, Ford RM, Cummings PT. Mathematical models for motile bacterial transport in cylindrical tubes. J Theor Biol 1998, 195:481-504.

104. Chen KC, Ford RM, Cummings PT. Perturbation expansion of Alt's cell balance equations reduces to Segel's one-dimensional equations for shallow chemoattractant gradient. SIAM J Appl Math 1998, 59:35-57.

105. Chen KC, Ford RM, Cummings PT. Spatial effect of tumbling frequencies for motile bacteria on cell ball equations. Chem Eng Sci 1999, 54:593-617.

106. Setayeshgar S, Gear CW, Othmer HG, Kevrekidis IG. Application of coarse integration to bacterial chemotaxis. Multiscale Model Simul 2005, 4:307-327.
107. Erban R, Othmer HG. From individual to collective behaviour in bacterial chemotaxis. SIAM J Appl Math 2004, 65:361-391.

108. Erban R, Othmer HG. From signal transduction to spatial pattern formation in E. coli: a paradigm for multiscale modelling in biology. Mol Syst Biol 2005, 3:362-394.

109. de Gennes PG. Chemotaxis: the role of internal delays. Eur Biophys J 2004, 33:691-693.

110. Clark DA, Grant LC. The bacterial chemotactic response reflects a compromise between transient and steady-state behaviour. Proc Nat Acad Sci 2005, 102:9150-9155.

111. Celani A, Vergassola M. Bacterial strategies for chemotaxis response. Proc Nat Acad Sci 2010, 107:1391-1396.

112. Bray D, Levin MD, Lipkow K. The chemotactic behavior of computer-based surrogate bacteria. Curr Biol 2007, 17:12-19.

113. Ben-Jacob E, Schochet O, Tenenbaum A, Cohen I, Czirok A, Vicsek T. Generic modelling of cooperative growth patterns in bacterial colonies. Nature 1994, 368:46-49.

114. Ben-Jacob E, Cohen I, Schochet O. Complex bacterial patterns. Nature 1995, 373:566-569.

115. Berg HC. Symmetries in bacterial motility. Proc Nat Acad Sci 1996, 93:14225-14228.

116. Budrene E, Berg H. Complex patterns formed by motile cells of Escherichia coli. Nature 1991, 349:630-633.

117. Keller EF, Segel LA. Initiation of slime mold aggregation viewed as an instability. J Theor Biol 1970, 26:399-415

118. Brenner MP, Levitov LS, Budrene EO. Physical mechanisms for chemotactic pattern formation by bacteria. Biophys J 1998, 74:1677-1693.

119. Hillesdon AJ, Pedley TJ, Kessler JO. The development of concentration gradients in a suspension of chemotactic bacteria. Bull Math Biol 1995, 57:299-334.

120. Zhu M, Murray JD. Parameter domains for generating spatial pattern: a comparison of reaction-diffusion and cell-chemotaxis models. Int J Bifurc Chaos 1995, $5: 1503-1524$

121. Leake MC. Shining the spotlight on functional molecular complexes: the new science of single-molecule cell biology. Commun Integr Biol 2010, 3:415-418.

122. Shoval O, Goentoro L, Hart Y, Mayo A, Sontag E, Alon U. Fold-change detection and scalar symmetry of sensory input fields. Proc Nat Acad Sci 2010, 107:15995-16000.

123. Lazova MD, Ahmed T, Bellomo D, Stocker R, Shimizu TS. Response rescaling in bacterial chemotaxis. Proc Nat Acad Sci USA 2011, 108:13870-13875. 